\title{
AN ITERATIVE ALGORITHM FOR SOLUTION OF CONTACT PROBLEMS OF BEAMS, PLATES, AND SHELLS
}

\author{
JAN AWREJCEWICZ AND ANTON V. KRYSKO
}

Received 1 June 2006; Accepted 16 July 2006

An iterative algorithm to solve efficiently one-sided interaction between two rectangular plates within the Kirchhoff hypothesis is proposed. Then a proof of convergence of this algorithm is given. The formulated theorem, proof, and five remarks exhibit advantages of our proposed novel approach.

Copyright (C) 2006 J. Awrejcewicz and A. V. Krysko. This is an open access article distributed under the Creative Commons Attribution License, which permits unrestricted use, distribution, and reproduction in any medium, provided the original work is properly cited.

\section{Introduction}

Nonlinear dynamics of beams, plates, and shells is exhibited in various engineering structures (buildings, bridges, tanks) and machines such as flight-vehicles, power plants, and various mechatronical devices.

In many engineering cases, an interaction of the multilayer mentioned construction members plays an important role, and hence it requires both careful mathematical modeling and analysis. The mentioned interaction is associated with one- and two-sided constraints creating a complex but challenging topic of research from the point of view of theory and application.

For instance, many investigations focus on the case when the deflection constraints are introduced along a beam length. This study possesses great importance, since various objects with more than three supports can be reduced to an analysis of the dynamical behavior of beams [4]. However, the given problem is not understood in full. In a majority of investigations devoted to this problem, ball-type supports are applied, and the axial massless springs are used as barriers [13]. The most popular models include a beam clamped on the end, and possessing either a concentrated mass [15] or a reduced torque on the second end [14].

In [10], complex vibrations of an Euler-Bernoulli beam with different types of nonlinearities are considered. An arbitrary beam clamping is considered, and deflection

Hindawi Publishing Corporation

Mathematical Problems in Engineering

Volume 2006, Article ID 71548, Pages 1-13

DOI 10.1155/MPE/2006/71548 
constraints (point barriers) are introduced in some beam points along its length. The influence of a constraint, as well as of the amplitude and frequency of excitation on the vibrations, is analyzed. Scenarios of the transition to chaos owing to the introduced nonlinearities are reported.

In [11], regular and chaotic vibrations together with bifurcations of flexible plate strips with nonsymmetric boundary conditions are investigated by the Bubnov-Galerkin method and a finite difference method of order $O\left(h^{4}\right)$. Special attention is paid to nonsymmetric boundary conditions. Lyapunov exponents are estimated via Bennetin's method. Some new examples of routes from regular to chaotic dynamics, and within chaotic dynamics, are illustrated and discussed. The phase transitions from chaos to hyperchaos, and a novel phenomenon of a shift from hyperchaos to another hyperhyper chaos are also reported.

In the work by Awrejcewicz et al. [3], a novel iteration procedure is proposed for dynamical problems, where in each time step a contacting plate zone are improved. Therefore, a zone and magnitude of a contact load are also improved. The effect of boundary conditions on externally driven vibrations of uncoupled two-layer plates, with the Kirchhoff hypothesis holding for each layer, is investigated.

Below, we consider mechanical one-sided interaction between two rectangular plates [2]. It is assumed that the plates are thin and their stress-strain state is governed by classical Kirchhoff's theory supplemented by physical nonlinearities introduced by the theory of small elastic-plastic deformations. We assume, in addition, that a contact pressure (normal to stress surface) is significantly less in comparison to normal stresses measured in cross-sections of a plate, and that the plates slip in contact zones in a free way.

Note that the choice of the classical theory of plates is motivated by an observation that an influence of transversal shear deformation on the stress-strain state and on the distribution of contact pressure is essentially less important than that of transversal clamping in a contact zone. The latter factor plays a key role in our investigations.

\section{Mathematical model}

Differential equations governing behavior of contacting plates have the forms

$$
\begin{aligned}
& A_{1} w_{1}(x, y)=q_{1}(x, y)-q_{k} \psi, \\
& A_{2} w_{2}(x, y)=q_{2}(x, y)+q_{k} \psi,
\end{aligned}
$$

where $w_{i}(x, y)$ are vector functions, $q_{i}(x, y)$ is vector of external load, $i$ is plate number measured in direction of a positive normal axis. Contact pressure being proportional to transversal clamping $w_{1}-h_{1}-w_{2}$ in a contact zone is as follows:

$$
q_{k}(x, y)=k \frac{E}{h}\left(w_{1}-h_{1}-w_{2}\right),
$$

and the function $\psi$ has the form

$$
\psi=\frac{\left[1+\operatorname{sign}\left(w_{1}-h_{1}-w_{2}\right)\right]}{2},
$$

where $h_{1}$ denotes clearance between plates. 
Observe that formula (2.2) holds for plates contact with the same values of $k$ and $h$. Contact problems of the Kirchhoff theory of plates are associated with Winkler-type coupling between clamping and contact pressure.

If the initial plates distribution (clearance function $h_{1}$ ) and the load are such that there is no contact after deformation, then $\psi \equiv 0$, and system (2.1) is uncoupled. Otherwise, system (2.1) contains coupled equations. Substituting (2.2) into (2.1), we have

$$
\begin{aligned}
& A_{1} w_{1}(x, y)=q_{1}(x, y)-k \frac{E}{h}\left(w_{1}-h_{1}-w_{2}\right) \psi, \\
& A_{2} w_{2}(x, y)=q_{2}(x, y)+k \frac{E}{h}\left(w_{1}-h_{1}-w_{2}\right) \psi .
\end{aligned}
$$

Eighth-order system (2.4) is further studied in the frame of the following boundary conditions:

(a) ball-type support

$$
\left.w\right|_{\partial \Omega}=\left.\frac{\partial^{2} w}{\partial n^{2}}\right|_{\partial \Omega}=0 ;
$$

(b) stiff clamping

$$
\left.w\right|_{\partial \Omega}=\left.\frac{\partial w}{\partial n}\right|_{\partial \Omega}=0
$$

Therefore, the mentioned equations define a physically and constructively nonlinear eighth-order problem.

Operator $A_{i}(i=1,2)$, for a physically nonlinear problem, has the following form [8]:

$$
\begin{aligned}
A_{i} w_{i}(x, y)= & \frac{\partial^{2}}{\partial x^{2}}\left(B_{11, i} \frac{\partial^{2} w_{i}}{\partial x^{2}}+B_{10, i} \frac{\partial^{2} w_{i}}{\partial y^{2}}\right)+\frac{\partial^{2}}{\partial y^{2}}\left(B_{10, i} \frac{\partial^{2} w_{i}}{\partial x^{2}}+B_{11, i} \frac{\partial^{2} w_{i}}{\partial y^{2}}\right) \\
& +2 \frac{\partial^{2}}{\partial x \partial y}\left(\left(B_{11, i}-B_{10, i}\right) \frac{\partial^{2} w_{i}}{\partial x \partial y}\right),
\end{aligned}
$$

where

$$
\begin{aligned}
& B_{m n, i}=\frac{1}{2}\left[\frac{E_{11, i}}{E_{01, i}}+(-1)^{n+1} \frac{E_{10, i}}{E_{00, i}}-E_{21, i}+(-1)^{n} E_{20, i}\right], \\
& E_{m n, i}=\int_{a_{i}(x, y)}^{b_{i}(x, y)} \frac{E_{i} z^{m}}{1+(-1)^{n} \lambda_{i}} d z, \quad(n=0.1, m=i=1,2),
\end{aligned}
$$

and where $z=a_{i}(x, y), z=b_{i}(x, y),(x, y) \in \Omega$ are the equations governing external plate surfaces, and allowing for introduction (during computations) of the variated thickness of plates.

Owing to application of the changeable elasticity parameters method [5], $E_{i}\left(x, y, z, e_{i}\right)$ and $v_{i}\left(x, y, z, e_{i}\right)$ are treated as parameters depending on the deformed plate states and 
4 An iterative algorithm for contact problems

they have the following forms:

$$
E_{i}=\frac{9 K_{1 i} G_{i}}{3 K_{1 i}+G_{i}}, \quad v_{i}=\frac{1}{2} \frac{3 K_{1 i}-2 G_{i}}{3 K_{1 i}+G_{i}},
$$

where $K_{1 i}=$ const. Recall that in the theory of deformation, the shear modulus is

$$
G_{i}=\frac{1}{3} \frac{\sigma_{i}^{(i)}\left(e_{i}^{(i)}\right)}{e_{i}^{(i)}}
$$

where $\sigma_{i}^{(i)}, e_{i}^{(i)}$ are the intensities of plate, stress and strain intensities $(i=1,2)$, respectively, and

$$
e_{x z}^{(i)}=-z \frac{\partial^{2} w_{i}}{\partial x^{2}}, \quad e_{y y}^{(i)}=-z \frac{\partial^{2} w_{i}}{\partial y^{2}}, \quad e_{x y}^{(i)}=-2 z \frac{\partial^{2} w_{i}}{\partial x \partial y} .
$$

In $(2.11), e_{z z}^{(i)}$ are defined by flat stress condition state of the form $\left(\sigma_{z z}=0\right)$ :

$$
e_{z z}^{(i)}=-\frac{\nu_{i}}{1-v_{i}}\left(e_{x x}^{(i)}+e_{y y}^{(i)}\right)
$$

System (2.4) is solved by the method of variational iterations (MVI). In order to solve the constructively nonlinear problem (2.4), one may apply an iterative process allowing to solve only one equation from system (2.4) on each loading step. The same approach in the case of geometrically nonlinear problems of shells has been proposed and applied in [6].

Application of the mentioned technique yields two-time reduction of the system in the case of two-layer package, and it yields $n$-times order reduction in the case of a package composed of $n$ layers. Iterative procedure has the following forms:

$$
\begin{aligned}
& A_{1}\left(w_{1}^{(n)}\right)+k \frac{E}{h} \psi_{(n-1)} w_{1}^{(n)}=q_{1}(x, y)+k \frac{E}{h}\left(h_{1}+w_{2}^{(n-1)}\right) \psi_{(n-1)}, \\
& A_{2}\left(w_{2}^{(n)}\right)+k \frac{E}{h} \psi_{(n-1)} w_{2}^{(n)}=q_{2}(x, y)+k \frac{E}{h}\left(w_{1}^{(n-1)}-h_{1}\right) \psi_{(n-1)} .
\end{aligned}
$$

One has to attach to system (2.13) the corresponding boundary conditions for the $i$ th plate.

Let $R^{2}$ be the Euclidean surface with Descartes basis; $\Omega_{i} \in R^{2}$ is the area in this surface with its boundary $\partial \Omega_{i}(i=1,2), \bar{\Omega}_{i}=\Omega_{i} \cup \partial \Omega_{i}(x, y) \in \Omega_{i}, \Omega^{*}$ is the subarea of $\Omega_{i}$, for all $i, \Omega^{*} \subseteq \Omega_{i}$, and $n_{i}$ is the external normal to $\partial \Omega_{i}$.

As it has been mentioned already, (2.13) with the associated boundary conditions will be solved using MVI [7, 9]. For a fixed contacting zone, both MVI and changeable elasticity parameters (CEPs) procedures are applied with a successive improvement of a contacting zone by simple iterative procedure. Next, the solving procedure is repeated, that is, we have three iterative procedures embedded in each other. Ordinary differential equations are reduced to algebraic ones by a finite difference method with accuracy of $O\left(\delta^{2}\right)$, where $\delta$ is the mesh step. Algebraic equations are solved by the Gauss procedure on each time step. 


\section{Proof of convergence of iterative algorithms}

We are going to prove the convergence of iterative algorithms used to solve contact problems of freely coupled plates within Kirchhoff's hypotheses. Next, we consider 3D plates construction consisting of contacting plates prescribed by Kirchhoff's hypotheses, that is, system (2.4) with an account of (2.7) has the following forms:

$$
\begin{gathered}
A_{1}\left(w_{1}(x, y)\right)+k \frac{E}{h} w_{1} \psi(x, y)=q_{1}+k \frac{E}{h}\left(w_{2}+h_{1}\right) \psi(x, y), \\
A_{2}\left(w_{2}(x, y)\right)+k \frac{E}{h} w_{2} \psi(x, y)=q_{2}(1-\psi(x, y))+k \frac{E}{h}\left(w_{1}-h_{1}\right) \psi(x, y),
\end{gathered}
$$

with the associated boundary conditions

$$
\left.w_{i}\right|_{\partial \Omega_{i}}=\left.\frac{\partial w_{i}}{\partial n_{i}}\right|_{\partial \Omega_{i}}=0 \quad(i=1,2),
$$

and with the function defining the contacting plates zone $\Omega^{*}$ of the form

$$
\psi(x, y)= \begin{cases}1, & (x, y) \in \Omega^{*}, \\ 0, & (x, y) \notin \Omega^{*},\end{cases}
$$

where $q_{1}(x, y)$ and $q_{2}(x, y)$ are the functions of external loads acting on the first and second contacting plates, respectively; $\|\cdot\|_{A}$ is the norm in the normalized space $A ;(\cdot, \cdot)_{B}$ denotes scalar product in the Hilbert space $B$ (notation of functional spaces corresponds to that used in [12]).

In order to solve problems (3.1) and (3.2), the following iterative algorithm is applied:

$$
\begin{gathered}
A_{1}\left(w_{1}^{(n+1)}(x, y)\right)+k \frac{E}{h} w_{1}^{(n+1)} \psi(x, y)=q_{1}+k \frac{E}{h}\left(w_{2}^{(n)}+h_{1}\right) \psi(x, y), \\
A_{2}\left(w_{2}^{(n+1)}(x, y)\right)+k \frac{E}{h} w_{2}^{(n)} \psi(x, y)=q_{2}(1-\psi(x, y))+k \frac{E}{h}\left(w_{1}^{(n)}-h_{1}\right) \psi(x, y), \\
\left.w_{1}^{(n+1)}\right|_{\partial \Omega_{1}}=\left.\frac{\partial w_{1}^{(n+1)}}{\partial n_{1}}\right|_{\partial \Omega_{1}}=0,\left.\quad w_{2}^{(n+1)}\right|_{\partial \Omega_{2}}=\left.\frac{\partial w_{2}^{(n+1)}}{\partial n_{2}}\right|_{\partial \Omega_{2}}=0 .
\end{gathered}
$$

The following theorem holds.

Theorem 3.1. Let $\Omega_{i}(i=1,2)$ be bounded areas with the boundaries $\partial \Omega_{i}$ that satisfy Sobolev's embedding theorem conditions [12], let $\Omega^{*}$ be the measurable space, let $q_{i}(x, y) \subseteq$ $L_{2}\left(\Omega_{i}\right)$, and let there be real constants $c_{i}>0, D_{i}>0$ such that

$$
\begin{aligned}
D_{1}\left\|\Delta\left(w_{i}\right)\right\|_{L_{2}\left(\Omega^{*}\right)}^{2} & \left(B_{11, i} \frac{\partial^{2} w_{i}}{\partial x^{2}}+B_{10, i} \frac{\partial^{2} w_{i}}{\partial y^{2}}, \frac{\partial^{2} w_{i}}{\partial x^{2}}\right)_{L_{2}\left(\Omega_{2}\right)}+\left(B_{10, i} \frac{\partial^{2} w_{i}}{\partial x^{2}}+B_{11, i} \frac{\partial^{2} w_{i}}{\partial y^{2}}, \frac{\partial^{2} w_{i}}{\partial y^{2}}\right)_{L_{2}\left(\Omega_{2}\right)} \\
+ & {\left[\left(B_{11, i}+B_{10, i}\right) \frac{\partial^{2} w_{i}}{\partial x \partial y}, \frac{\partial^{2} w_{i}}{\partial x \partial y}\right]_{L_{2}\left(\Omega_{2}\right)} \leq c_{i}\left\|\Delta\left(w_{i}\right)\right\|_{L_{2}\left(\Omega_{i}\right)}^{2} }
\end{aligned}
$$


6 An iterative algorithm for contact problems

Then

(1) for all $n, w_{i}^{(n)} \in W_{2}^{4}\left(\Omega_{i}\right) \cap \dot{W}_{2}^{2}\left(\Omega_{i}\right), i=1,2$;

(2) there are functions $w_{i}^{*}(x, y) \in \dot{W}_{2}^{2}\left(\Omega_{i}\right), i=1,2$, being solutions of problems (3.1), (3.2), and $\lim _{n \rightarrow \infty}\left\|w_{i}^{(n)}-w_{i}^{*}\right\|_{W_{2}^{2}\left(\Omega_{i}\right)}=0$.

Proof. Only fundamental proof steps will be given. The conclusion of the first theorem follows from the theory of solvability of elliptic equations [12] assuming initial approximations $w_{i}^{0} \in L_{2}\left(\Omega_{i}\right), i=1,2$.

The conclusion of the second theorem proves an existence of a general solution to problems (3.1) and (3.2) in the space $\dot{W}_{2}^{2}\left(\Omega_{1}\right) \times \dot{W}_{2}^{2}\left(\Omega_{2}\right)$ and a strong convergence of a sequence of approximating solutions $\left\{w_{i}^{(n)}\right\}$ to exactly one $w_{i}^{*}$ with respect to the space norm $\dot{W}_{2}^{2}\left(\Omega_{i}\right), i=1,2$. In order to prove the second conclusion, the following operations should be applied:

(1) functions $w_{i}^{(n)}(i=1,2)$ are computed from (3.5);

(2) first equation of the obtained system is multiplied by $\left(w_{1}^{(n+1)}-w_{1}^{(n)}\right)$, whereas second equation is multiplied by $\left(w_{2}^{(n+1)}-w_{2}^{(n)}\right)$;

(3) again, the first equation is integrated in the space $\Omega_{1}\left(\Omega_{2}\right)$. As a result, after application of the Green formula, one gets

$$
\begin{aligned}
& \left(B_{10,1} \frac{\partial^{2}\left(w_{1}^{(n+1)}-w_{1}^{(n)}\right)}{\partial x^{2}}+B_{10,1} \frac{\partial^{2}\left(w_{1}^{(n+1)}-w_{1}^{(n)}\right)}{\partial y^{2}}, \frac{\partial^{2}\left(w_{1}^{(n+1)}-w_{1}^{(n)}\right)}{\partial x^{2}}\right)_{L_{2}\left(\Omega_{2}\right)} \\
& +\left(B_{10,1} \frac{\partial^{2}\left(w_{1}^{(n+1)}-w_{1}^{(n)}\right)}{\partial x^{2}}+B_{11,1} \frac{\partial^{2}\left(w_{1}^{(n+1)}-w_{1}^{(n)}\right)}{\partial y^{2}}, \frac{\partial^{2}\left(w_{1}^{(n+1)}-w_{1}^{(n)}\right)}{\partial x^{2}}\right)_{L_{2}\left(\Omega_{2}\right)} \\
& +\left(\left[B_{11,1}-B_{10,1}\right] \frac{\partial^{2}\left(w_{1}^{(n+1)}-w_{1}^{(n)}\right)}{\partial x \partial y}, \frac{\partial^{2}\left(w_{1}^{(n+1)}-w_{1}^{(n)}\right)}{\partial x \partial y}\right)_{L_{2}\left(\Omega_{1}\right)} \\
& +\frac{k E}{h}\left(\psi(x, y)\left(w_{1}^{(n+1)}-w_{1}^{(n)}\right),\left(w_{1}^{(n+1)}-w_{1}^{(n)}\right)\right)_{L_{2}\left(\Omega_{1}\right)} \\
& =\frac{k E}{h}\left(\psi(x, y)\left(w_{2}^{(n)}-w_{2}^{(n-1)}\right),\left(w_{1}^{(n+1)}-w_{1}^{(n)}\right)\right)_{L_{2}\left(\Omega_{1}\right)} \text {, } \\
& \left(B_{11,2} \frac{\partial^{2}\left(w_{2}^{(n+1)}-w_{2}^{(n)}\right)}{\partial x^{2}}+B_{10,2} \frac{\partial^{2}\left(w_{2}^{(n+1)}-w_{2}^{(n)}\right)}{\partial y^{2}}, \frac{\partial^{2}\left(w_{1}^{(n+1)}-w_{1}^{(n)}\right)}{\partial x^{2}}\right)_{L_{2}\left(\Omega_{2}\right)} \\
& +\left(B_{10,2} \frac{\partial^{2}\left(w_{2}^{(n+1)}-w_{2}^{(n)}\right)}{\partial x^{2}}+B_{11,2} \frac{\partial^{2}\left(w_{2}^{(n+1)}-w_{2}^{(n)}\right)}{\partial y^{2}}, \frac{\partial^{2}\left(w_{2}^{(n+1)}-w_{2}^{(n)}\right)}{\partial y^{2}}\right)_{L_{2}\left(\Omega_{2}\right)} \\
& +\left(\left[B_{11,2}-B_{10,2}\right] \frac{\partial^{2}\left(w_{2}^{(n+1)}-w_{2}^{(n)}\right)}{\partial x \partial y}, \frac{\partial^{2}\left(w_{2}^{(n+1)}-w_{2}^{(n)}\right)}{\partial x \partial y}\right)_{L_{2}\left(\Omega_{2}\right)}
\end{aligned}
$$




$$
\begin{aligned}
& +\frac{k E}{h}\left(\psi(x, y)\left(w_{2}^{(n+1)}-w_{2}^{(n)}\right),\left(w_{2}^{(n+1)}-w_{2}^{(n)}\right)\right)_{L_{2}\left(\Omega_{2}\right)} \\
= & \frac{k E}{h}\left(\psi(x, y)\left(w_{1}^{(n)}-w_{1}^{(n-1)}\right)\right) .
\end{aligned}
$$

Owing to the definition of functions $\psi(x, y),(3.8)$ assume the form

$$
\begin{aligned}
& \left(B_{11,1} \frac{\partial^{2}\left(w_{1}^{(n+1)}-w_{1}^{(n)}\right)}{\partial x^{2}}+B_{10,1} \frac{\partial^{2}\left(w_{1}^{(n+1)}-w_{1}^{(n)}\right)}{\partial y^{2}}, \frac{\partial^{2}\left(w^{(n+1)}-w^{(n)}\right)}{\partial x^{2}}\right)_{L_{2}\left(\Omega_{1}\right)} \\
& +\left(B_{10,1} \frac{\partial^{2}\left(w_{1}^{(n+1)}-w_{1}^{(n)}\right)}{\partial x^{2}}+B_{11,1} \frac{\partial^{2}\left(w_{1}^{(n+1)}-w_{1}^{(n)}\right)}{\partial y^{2}}, \frac{\partial^{2}\left(w_{1}^{(n+1)}-w_{2}^{(n)}\right)}{\partial y^{2}}\right)_{L_{2}\left(\Omega_{1}\right)} \\
& +\left(\left[B_{11,1}-B_{10,1}\right] \frac{\partial^{2}\left(w_{1}^{(n+1)}-w_{1}^{(n)}\right)}{\partial x \partial y}, \frac{\partial^{2}\left(w_{1}^{(n+1)}-w_{1}^{(n)}\right)}{\partial x \partial y}\right)_{L_{2}\left(\Omega_{1}\right)} \\
& +\frac{k E}{h}\left\|\left(w_{1}^{(n+1)}-w_{1}^{(n)}\right)\right\|_{L_{2}\left(\Omega^{*}\right)}^{2}=\frac{k E}{h}\left(\left(w_{2}^{(n)}-w_{2}^{(n-1)}\right),\left(w_{1}^{(n+1)}-w_{1}^{(n)}\right)\right)_{L_{2}\left(\Omega^{*}\right)^{\prime}} \\
& \left(B_{11,2} \frac{\partial^{2}\left(w_{2}^{(n+1)}-w_{2}^{(n)}\right)}{\partial x^{2}}+B_{10,2} \frac{\partial^{2}\left(w_{2}^{(n+1)}-w_{2}^{(n)}\right)}{\partial y^{2}}, \frac{\partial^{2}\left(w_{1}^{(n+1)}-w_{1}^{(n)}\right)}{\partial x^{2}}\right)_{L_{2}\left(\Omega_{2}\right)} \\
& +\left(B_{10,2} \frac{\partial^{2}\left(w_{2}^{(n+1)}-w_{2}^{(n)}\right)}{\partial x^{2}}+B_{11,2} \frac{\partial^{2}\left(w_{2}^{(n+1)}-w_{2}^{(n)}\right)}{\partial y^{2}}, \frac{\partial^{2}\left(w_{2}^{(n+1)}-w_{2}^{(n)}\right)}{\partial y^{2}}\right)_{L_{2}\left(\Omega_{2}\right)} \\
& +\frac{k E}{h}\left\|\left(w_{2}^{(n+1)}-w_{2}^{(n)}\right)\right\|_{L_{2}\left(\Omega^{*}\right)}^{2}=\frac{k E}{h}\left(\left(w_{1}^{(n)}-w_{1}^{(n-1)}\right),\left(w_{2}^{(n+1)}-w_{2}^{(n)}\right)\right)_{L_{2}\left(\Omega^{*}\right)}
\end{aligned}
$$

Applying Young's inequality [12], (3.9), and the theorem condition, we have

$$
\begin{aligned}
& D_{1} \| \Delta\left(w_{1}^{(n+1)}-w_{1}^{(n)}\right)\left\|_{L_{2}\left(\Omega^{*}\right)}^{2}+\frac{k E}{h}\right\|\left(w_{1}^{(n+1)}-w_{1}^{(n)}\right) \|_{L_{2}\left(\Omega^{*}\right)}^{2} \\
& \leq \frac{k E}{2 h}\left\|\left(w_{2}^{(n)}-w_{2}^{(n-1)}\right)\right\|_{L_{2}\left(\Omega^{*}\right)}^{2}+\frac{k E}{2 h}\left\|\left(w_{1}^{(n+1)}-w_{1}^{(n)}\right)\right\|_{L_{2}\left(\Omega^{*}\right)}^{2}, \\
& D_{2}\left\|\Delta\left(w_{2}^{(n+1)}-w_{2}^{(n)}\right)\right\|_{L_{2}\left(\Omega_{2}\right)}^{2}+\frac{k E}{h}\left\|\left(w_{2}^{(n+1)}-w_{2}^{(n)}\right)\right\|_{L_{2}\left(\Omega^{*}\right)}^{2} \\
& \quad \leq \frac{k E}{2 h}\left\|\left(w_{1}^{(n)}-w_{1}^{(n-1)}\right)\right\|_{L_{2}\left(\Omega^{*}\right)}^{2}+\frac{k E}{2 h}\left\|\left(w_{2}^{(n+1)}-w_{2}^{(n)}\right)\right\|_{L_{2}\left(\Omega^{*}\right)}^{2}
\end{aligned}
$$


8 An iterative algorithm for contact problems

and finally we get

$$
\begin{aligned}
& D_{1}\left\|\Delta\left(w_{1}^{(n+1)}-w_{1}^{(n)}\right)\right\|_{L_{2}\left(\Omega^{*}\right)}^{2}+\frac{k E}{2 h}\left\|\left(w_{2}^{(n)}-w_{2}^{(n-1)}\right)\right\|_{L_{2}\left(\Omega^{*}\right)}^{2} \leq \frac{k E}{2 h}\left\|\left(w_{2}^{(n+1)}-w_{2}^{(n)}\right)\right\|_{L_{2}\left(\Omega^{*}\right)}^{2} \\
& D_{2}\left\|\Delta\left(w_{1}^{(n+1)}-w_{1}^{(n)}\right)\right\|_{L_{2}\left(\Omega^{*}\right)}^{2}+\frac{k E}{2 h}\left\|\left(w_{2}^{(n+1)}-w_{2}^{(n)}\right)\right\|_{L_{2}\left(\Omega^{*}\right)}^{2} \leq \frac{k E}{2 h}\left\|\left(w_{1}^{(n)}-w_{1}^{(n-1)}\right)\right\|_{L_{2}\left(\Omega^{*}\right)}^{2}
\end{aligned}
$$

Inequalities (3.11) are rewritten in the forms

$$
\begin{aligned}
& D_{1}\left\|\Delta\left(w_{1}^{(n+1)}-w_{1}^{(n)}\right)\right\|_{L_{2}\left(\Omega^{*}\right)}^{2}+\frac{k E}{2 h}\left\|\left(w_{1}^{(n+1)}-w_{1}^{(n)}\right)\right\|_{L_{2}\left(\Omega^{*}\right)}^{2} \\
& \quad \leq(1-\alpha) \frac{k E}{2 h}\left\|\left(w_{2}^{(n)}-w_{2}^{(n-1)}\right)\right\|_{L_{2}\left(\Omega^{*}\right)}^{2}+\alpha \frac{k E}{2 h}\left\|\left(w_{2}^{(n)}-w_{2}^{(n-1)}\right)\right\|_{L_{2}\left(\Omega^{*}\right)}^{2}, \\
& D_{2}\left\|\Delta\left(w_{2}^{(n+1)}-w_{2}^{(n)}\right)\right\|_{L_{2}\left(\Omega_{2}\right)}^{2}+\frac{k E}{2 h}\left\|\left(w_{2}^{(n+1)}-w_{2}^{(n)}\right)\right\|_{L_{2}\left(\Omega^{*}\right)}^{2} \\
& \quad \leq(1-\alpha) \frac{k E}{2 h}\left\|\left(w_{1}^{n}-w_{1}^{(n-1)}\right)\right\|_{L_{2}\left(\Omega^{*}\right)}^{2}+\alpha \frac{k E}{2 h}\left\|\left(w_{1}^{(n)}-w_{1}^{(n-1)}\right)\right\|_{L_{2}\left(\Omega^{*}\right)}^{2},
\end{aligned}
$$

where $\alpha \in R^{1}, 0<\alpha<1$.

Next, we apply the Friedrichs inequality [1] in the following form. One may find a constant $c_{i} \in R^{1}$ such that for all $f(x, y) \in \dot{W}_{2}^{2}\left(\Omega_{i}\right), i=1,2$, the following inequalities hold:

$$
\|f\|_{L_{2}\left(\Omega^{*}\right)}^{2} \leq c_{i}\|\Delta f\|_{L_{2}\left(\Omega^{*}\right)}^{2} \leq c_{i}\|\Delta f\|_{L_{2}(\Omega)}^{2}, \quad i=1,2
$$

under that assumption that $\Omega^{*} \subseteq \Omega_{i}$ and that it satisfies conditions of application of Friedrichs inequality. Owing to (3.14), the inequalities (3.12) and (3.13) have the following forms:

$$
\begin{aligned}
& D_{1}\left\|\Delta\left(w_{1}^{(n+1)}-w_{1}^{(n)}\right)\right\|_{L_{2}\left(\Omega_{i}\right)}^{2}+\frac{k E}{2 h}\left\|\left(w_{1}^{(n+1)}-w_{1}^{(n)}\right)\right\|_{L_{2}\left(\Omega^{*}\right)}^{2} \\
& \quad \leq(1-\alpha) \frac{k E}{2 h}\left\|\left(w_{2}^{(n)}-w_{2}^{(n-1)}\right)\right\|_{L_{2}\left(\Omega^{*}\right)}^{2}+\frac{\alpha C_{2} k E}{D_{2} 2 h} D_{1}\left\|\Delta\left(w_{1}^{(n+1)}-w_{1}^{(n)}\right)\right\|_{L_{2}(\Omega)}^{2}, \\
& D_{2}\left\|\Delta\left(w_{2}^{(n+1)}-w_{2}^{(n)}\right)\right\|_{L_{2}\left(\Omega_{2}\right)}^{2}+\frac{k E}{2 h}\left\|\left(w_{2}^{(n+1)}-w_{2}^{(n)}\right)\right\|_{L_{2}\left(\Omega^{*}\right)}^{2} \\
& \quad \leq(1-\alpha) \frac{k E}{2 h}\left\|\left(w_{1}^{(n)}-w_{1}^{(n-1)}\right)\right\|_{L_{2}\left(\Omega^{*}\right)}^{2}+\frac{\alpha C_{1} k E}{D_{1} 2 h} D_{1}\left\|\Delta\left(w_{1}^{(n+1)}-w_{1}^{(n)}\right)\right\|_{L_{2}(\Omega)}^{2}
\end{aligned}
$$


We choose $\alpha$ in inequalities (3.15) from the following condition:

$$
\frac{\alpha C_{2} k E}{2 h D_{2}}<1, \quad \frac{\alpha C_{1} k E}{2 h D_{1}}<1, \quad 0<\alpha<1,
$$

and we take

$$
\rho=\max \left\{(1-\alpha), \frac{\alpha C_{2} k E}{2 h D_{2}}, \frac{\alpha C_{1} k E}{2 h D_{1}}\right\}
$$

It follows immediately that $0<\rho<1$.

Now, inequalities (3.15) can have the following forms:

$$
\begin{aligned}
& D_{1}\left\|\Delta\left(w_{1}^{(n+1)}-w_{1}^{(n)}\right)\right\|_{L_{2}\left(\Omega_{i}\right)}^{2}+\frac{k E}{2 h}\left\|\left(w_{1}^{(n+1)}-w_{1}^{(n)}\right)\right\|_{L_{2}\left(\Omega^{*}\right)}^{2} \\
& \quad \leq \rho\left\{D_{2}\left\|\Delta\left(w_{2}^{(n)}-w_{2}^{(n-1)}\right)\right\|_{L_{2}\left(\Omega_{2}\right)}^{2}+\frac{k E}{2 h}\left\|\left(w_{2}^{(n)}-w_{2}^{(n-1)}\right)\right\|_{L_{2}\left(\Omega^{*}\right)}^{2}\right\}, \\
& D_{2}\left\|\Delta\left(w_{2}^{(n+1)}-w_{2}^{(n)}\right)\right\|_{L_{2}\left(\Omega_{2}\right)}^{2}+\frac{k E}{2 h}\left\|\left(w_{2}^{(n+1)}-w_{2}^{(n)}\right)\right\|_{L_{2}\left(\Omega^{*}\right)}^{2} \\
& \quad \leq \rho\left\{D_{1}\left\|\Delta\left(w_{1}^{(n)}-w_{1}^{(n-1)}\right)\right\|_{L_{2}\left(\Omega_{1}\right)}^{2}+\frac{k E}{2 h}\left\|\left(w_{1}^{(n)}-w_{1}^{(n-1)}\right)\right\|_{L_{2}\left(\Omega^{*}\right)}^{2}\right\} .
\end{aligned}
$$

Matching inequalities (3.18) yields

$$
\begin{gathered}
\left\{D_{1}\left\|\Delta\left(w_{1}^{(n+1)}-w_{1}^{(n)}\right)\right\|_{L_{2}\left(\Omega_{1}\right)}^{2}+D_{2}\left\|\Delta\left(w_{2}^{(n+1)}-w_{2}^{(n)}\right)\right\|_{L_{2}\left(\Omega_{2}\right)}^{2}\right. \\
\left.+\frac{k E}{2 h}\left\|\left(w_{1}^{(n+1)}-w_{1}^{(n)}\right)\right\|_{L_{2}\left(\Omega^{*}\right)}^{2}+\frac{k E}{2 h}\left\|\left(w_{2}^{(n+1)}-w_{2}^{(n)}\right)\right\|_{L_{2}\left(\Omega^{*}\right)}^{2}\right\} \\
\leq \rho\left\{D_{1}\left\|\Delta\left(w_{1}^{(n)}-w_{1}^{(n-1)}\right)\right\|_{L_{2}\left(\Omega_{1}\right)}^{2}+D_{2}\left\|\Delta\left(w_{2}^{(n)}-w_{2}^{(n-1)}\right)\right\|_{L_{2}\left(\Omega_{1}\right)}^{2}\right. \\
\left.+\frac{k E}{2 h}\left\|\left(w_{1}^{(n)}-w_{1}^{(n-1)}\right)\right\|_{L_{2}\left(\Omega^{*}\right)}^{2}+\frac{k E}{2 h}\left\|\left(w_{2}^{(n)}-w_{2}^{(n-1)}\right)\right\|_{L_{2}\left(\Omega^{*}\right)}^{2}\right\}
\end{gathered}
$$

or

$$
\left\|\bar{w}^{(n+1)}-\bar{w}^{(n)}\right\|_{H}^{2} \leq \rho\left\|\bar{w}^{(n)}-\bar{w}^{(n-1)}\right\|_{H}^{2},
$$

where $\bar{w}^{(n)}=\left(w_{1}^{(n)}, w_{2}^{(n)}\right), H$ is the normalized space with the norm equivalent to that of space $\dot{W}_{2}^{2}\left(\Omega_{1}\right) \times \dot{W}_{2}^{2}\left(\Omega_{2}\right)$ and defined by left (or right) part of inequality (3.19).

Inequality (3.20) yields

$$
\begin{gathered}
\left\|\bar{w}^{(n+1)}-\bar{w}^{(n)}\right\|_{H} \leq \rho\left\|\bar{w}^{(n)}-\bar{w}^{(n-1)}\right\|_{H}, \quad \rho_{1}=\sqrt{\rho}<1, \\
\left\|\bar{w}^{(n+1)}-\bar{w}^{(n)}\right\|_{H} \leq \theta \rho_{1}^{n},
\end{gathered}
$$


where

$$
\theta=\left\|\bar{w}^{(1)}-\bar{w}^{0}\right\|_{H}
$$

Hence, for any natural $\rho$, one obtains

$$
\begin{aligned}
\left\|\bar{w}^{(n+p)}-\bar{w}^{(n)}\right\|_{H} & \leq\left\|\bar{w}^{(n+p)}-\bar{w}^{(n+p-1)}\right\|_{H}+\left\|\bar{w}^{(n+p-1)}-\bar{w}^{(n+p-2)}\right\|_{H}+\left\|\bar{w}^{(n+1)}-\bar{w}^{(n)}\right\|_{H} \\
& \leq \theta \rho_{1}^{(n+p-1)}+\theta \rho_{1}^{(n+p-2)}+\cdots+\theta \rho_{1}^{(n)}=\frac{\theta\left(\rho_{1}^{(n)}+\rho_{1}^{(n+p)}\right)}{1-\rho_{1}} \leq \frac{\theta \rho_{1}^{(n)}}{1-\rho_{1}} .
\end{aligned}
$$

Inequality (3.23) implies fundamentality of the sequence $\left\{\bar{w}^{(n)}\right\}$, and owing to completeness of $H$, also convergence $\left\{\bar{w}^{(n)}\right\}$ in $H$ to the function $\bar{w}^{*}=\left(w_{1}^{*}, w_{2}^{*}\right)$ holds.

Therefore, owing to equivalence of the norms in spaces $H$ and $\dot{W}_{2}^{2}\left(\Omega_{1}\right) \times \dot{W}_{2}^{2}\left(\Omega_{2}\right)$, one gets

$$
\lim _{n \rightarrow \infty}\left\|w_{i}^{n}-w_{i}^{*}\right\|_{\dot{W}_{2}^{2}\left(\Omega_{i}\right)}=0, \quad i=1,2 .
$$

Systems (3.5) and (3.6) can be presented in the following integral forms:

$$
\begin{aligned}
& \left(B_{11,1} \frac{\partial^{2} w_{1}^{(n+1)}}{\partial x^{2}}+B_{10,1} \frac{\partial^{2} w_{1}^{(n+1)}}{\partial y^{2}}, \frac{\partial^{2} \varphi_{1}}{\partial x^{2}}\right)_{L_{2}\left(\Omega_{1}\right)}+\left(B_{10,1} \frac{\partial^{2} w_{1}^{(n+1)}}{\partial x^{2}}+B_{11,1} \frac{\partial^{2} w_{1}^{(n+1)}}{\partial y^{2}}, \frac{\partial^{2} \varphi_{1}}{\partial y^{2}}\right)_{L_{2}\left(\Omega_{1}\right)} \\
& +\left(\left[B_{11,1}-B_{10,1}\right] \frac{\partial^{2} w_{1}^{(n+1)}}{\partial x \partial y} \frac{\partial^{2} \varphi_{1}}{\partial x \partial y}\right)_{L_{2}\left(\Omega_{1}\right)}+\frac{k E}{h}\left(\psi(x, y) w_{1}^{(n+1)}, \varphi_{1}\right)_{L_{2}\left(\Omega_{1}\right)} \\
& =\left(q_{1} \varphi_{1}\right)_{L_{2}\left(\Omega_{1}\right)}+\frac{k E}{h}\left(\psi(x, y)\left[w_{1}^{(n)}+h_{1}\right], \varphi_{1}\right)_{L_{2}\left(\Omega^{*}\right)}, \\
& \left(B_{11,2} \frac{\partial^{2} w_{1}^{(n+1)}}{\partial x^{2}}+B_{10,2} \frac{\partial^{2} w_{2}^{(n+1)}}{\partial y^{2}}, \frac{\partial^{2} \varphi_{2}}{\partial x^{2}}\right)_{L_{2}\left(\Omega_{2}\right)}+\left(B_{10,2} \frac{\partial^{2} w_{2}^{(n+1)}}{\partial x^{2}}+B_{11,2} \frac{\partial^{2} w_{2}^{(n+1)}}{\partial y^{2}}, \frac{\partial^{2} \varphi_{2}}{\partial y^{2}}\right)_{L_{2}\left(\Omega_{2}\right)} \\
& +\left(\left[B_{11,2}-B_{10,2}\right] \frac{\partial^{2} w_{2}^{(n+1)}}{\partial x \partial y} \frac{\partial^{2} \varphi_{2}}{\partial x \partial y}\right)_{L_{2}\left(\Omega_{2}\right)}+\frac{k E}{h}\left(\psi(x, y) w_{2}^{(n+1)}, \varphi_{2}\right)_{L_{2}\left(\Omega_{2}\right)} \\
& =\left(q_{2}[1-\psi(x, y)], \varphi_{2}\right)_{L_{2}\left(\Omega_{2}\right)}+\frac{k E}{h}\left(\psi(x, y)\left[w_{1}^{(n)}+h_{1}\right], \varphi_{1}\right)_{L_{2}\left(\Omega^{*}\right)} \\
& \forall \varphi_{1} \in \dot{W}_{2}^{2}\left(\Omega_{1}\right), \forall \varphi_{2} \in \dot{W}_{2}^{2}\left(\Omega_{2}\right) .
\end{aligned}
$$


Owing to condition (3.12), one may apply a limiting transition for $n \rightarrow \infty$ in (3.13) and (3.14) to get

$$
\begin{aligned}
& \left(B_{11,1} \frac{\partial^{2} w_{1}^{*}}{\partial x^{2}}+B_{10,1} \frac{\partial^{2} w_{1}^{*}}{\partial y^{2}}, \frac{\partial^{2} \varphi_{1}}{\partial x^{2}}\right)_{L_{2}\left(\Omega_{1}\right)}+\left(B_{10,1} \frac{\partial^{2} w_{1}^{*}}{\partial x^{2}}+B_{11,1} \frac{\partial^{2} w_{1}^{*}}{\partial y^{2}}, \frac{\partial^{2} \varphi_{1}}{\partial y^{2}}\right)_{L_{2}\left(\Omega_{1}\right)} \\
& +\left(\left[B_{11,1}-B_{10,1}\right] \frac{\partial^{2} w_{1}^{*}}{\partial x \partial y} \frac{\partial^{2} \varphi_{1}}{\partial x \partial y}\right)_{L_{2}\left(\Omega_{1}\right)}+\frac{k E}{h}\left(\psi(x, y) w^{*}, \varphi_{1}\right)_{L_{2}\left(\Omega_{1}\right)} \\
& =\left(q_{1} \varphi_{1}\right)_{L_{2}\left(\Omega_{1}\right)}+\frac{k E}{h}\left(\psi(x, y)\left[w_{2}^{*}+h_{1}\right], \varphi_{1}\right)_{L_{2}\left(\Omega^{*}\right)}, \\
& \left(B_{11,2} \frac{\partial^{2} w_{1}^{*}}{\partial x^{2}}+B_{10,2} \frac{\partial^{2} w_{1}^{*}}{\partial y^{2}}, \frac{\partial^{2} \varphi_{1}}{\partial x^{2}}\right)_{L_{2}\left(\Omega_{2}\right)}+\left(B_{10,2} \frac{\partial^{2} w_{1}^{*}}{\partial x^{2}}+B_{11,2} \frac{\partial^{2} w_{1}^{*}}{\partial y^{2}}, \frac{\partial^{2} \varphi_{1}}{\partial y^{2}}\right)_{L_{2}\left(\Omega_{2}\right)} \\
& +\left(\left[B_{11,2}-B_{10,2}\right] \frac{\partial^{2} w_{1}^{*}}{\partial x \partial y} \frac{\partial^{2} \varphi_{1}}{\partial x \partial y}\right)_{L_{2}\left(\Omega_{2}\right)}+\frac{k E}{h}\left(\psi(x, y) w_{2}^{*}, \varphi_{2}\right)_{L_{2}\left(\Omega_{2}\right)} \\
& =\left(q_{2}[1-\psi(x, y)], \varphi_{2}\right)_{L_{2}\left(\Omega_{2}\right)}+\frac{k E}{h}\left(\psi(x, y)\left[w_{1}^{*}+h_{1}\right], \varphi_{1}\right)_{L_{2}\left(\Omega^{*}\right)},
\end{aligned}
$$

which proves the second conclusion of the theorem.

Remark 3.2. Convergence of the iterative algorithm is easily extended into the case of other boundary conditions. For instance, the proposed scheme of the proof remains valid when one or two contacting plates are ball-type supported.

Remark 3.3. The proposed iterative algorithm can be applied in the problems of contacting plates taking into account physical nonlinearities (matching with the method of elastic solution of Iliushin causes the scheme of proof to remain valid).

Remark 3.4. Convergence of the proposed iterative algorithm can be extended into solution of contact problems of 3D constructions composed of freely coupled plates within Timoshenko-type hypotheses for each plate, as well as for combined models, that is, when one of the layers is associated with Kirchhoff's hypothesis, and for other one the Timoshenko assumptions hold.

Remark 3.5. In relations (2.1), (2.4), (2.13), (3.1), and (3.5), $q_{i}=q_{i}^{0}-(y / g) h\left(\partial^{2} w_{i} / \partial t^{2}\right)-$ $\varepsilon_{i} h\left(\partial w_{i} / \partial t\right)$ denotes intensity of the given external loads, and inertia forces acting on the $i$ th plate are taken into account according to d'Alembert's principle; $\varepsilon_{i}$ is the coefficient of the mechanical property medium; $g$ is the acceleration of gravity; $\gamma$ is the unit material gravity coefficient; and $t$ is time.

Remark 3.6. The proposed iterative procedure is carried out on each time step. 


\section{Conclusions}

This paper is basically devoted to a rigorous proof of convergence of the iterative algorithm applied to solve contact problems of freely coupled plates within Kirchhoff's hypotheses. The main effort of our research is focused on the formulation of a theorem and its proof. It is expected that the given theorem may play an important role in various problems of beams, plates, and shells dynamics with both changeable contacting zones and elasticity of parameters.

\section{Acknowledgments}

Anton V. Krysko would like to acknowledge a financial support from the National Grant of Russia and hospitality of Prof. J. Awrejcewicz's department during this research.

\section{References}

[1] J. Awrejcewicz and V. A. Krysko, Nonclassical Thermoelastic Problems in Nonlinear Dynamics of Shells, Scientific Computation, Springer, Berlin, 2003.

[2] _ An iterative algorithm for solution of contact problems of beams, plates and shells, 5th EUROMECH Nonlinear Dynamics Conference (ENOC '05), Eindhoven, 2005, pp. 2477-2483, (CD Rom).

[3] J. Awrejcewicz, V. A. Krysko, and O. Ovsiannikova, Novel procedure to compute a contact zone magnitude of vibrations of two-layered uncoupled plates, Mathematical Problems in Engineering 2005 (2005), no. 4, 425-435.

[4] V. M. Baranovskiy and A. I. Mil'chenko, Deflection of statical indefinity of beams using method of complex acceptances, Chemical Petrol Machines Design 6 (1997), 1-9 (Russian).

[5] I. A. Birger, Some general methods of solution of problems of the theory of plasticity, Prikladnaya Matematika i Mekhanika 15 (1951), no. 6, 765-770 (Russian).

[6] V. V. Bochkarev and V. A. Krysko, On solution of geometrically nonlinear problems of plates, Izviestia Vuzov, Stroitel'stov i Architektura 10 (1981), 30-34 (Russian).

[7] L. V. Kantorovich, On convergence of the reduction into ordinary differential equation, Doklady Akademii Nauk SSSR 30 (1941), no. 7, 579-582 (Russian).

[8] V. A. Krysko, Nonlinear Statics and Dynamics of Non-Homogeneous Shells, Saratov State University Press, Saratov, 1976.

[9] _ Mathematical Models of Contacting Problems of Plates and Shells, Saratov State University Press, Saratov, 2000.

[10] V. A. Krysko and J. Awrejcewicz, On the vibration of the Euler-Bernoulli beam with clamped ends deflection constraints, International Journal of Bifurcation and Chaos in Applied Sciences and Engineering 15 (2005), no. 6, 1867-1878.

[11] V. A. Krysko, J. Awrejcewicz, and G. G. Narkaitis, Nonlinear vibration and characteristics of flexible plate-strips with non-symmetric boundary conditions, Communications in Nonlinear Science and Numerical Simulation 11 (2006), no. 1, 95-124.

[12] O. A. Ladyzhenskaya and I. N. Uraltseva, Linear and Quasi-Linear Equations of Elliptic Type, 2nd ed., Nauka, Moscow, 1973.

[13] K. Nagai, K. Kasuga, M. Kamada, T. Yamaguchi, and K. Tanifuji, Experiment on chaotic oscillations of a postbuckled reinforced beam constrained by an axial spring, Transactions of the Japan Society of Mechanical Engineers-Part C 41 (1998), no. 3, 563-569. 
[14] O. V. Vasenova and S. N. Fillipova, Solution to the problem on in plane rod bending using finite element method, Scientific Works of Moscow State University, Neft'iGaz 3 (1996), 59-68, in Russian.

[15] W. D. Zhu and C. D. Mote Jr., Dynamic modeling and optimal control of rotating Euler-Bernoulli beams, Transactions of the ASME. Journal of Dynamic Systems, Measurement and Control 119 (1997), no. 4, 802-808.

Jan Awrejcewicz: Department of Automatics and Biomechanics, Technical University of Łódź, 90-924 Łódź, Poland

E-mail address: awrejcew@p.lodz.pl

Anton V. Krysko: Department of Mathematics, Saratov State Technical University, 410054 Saratov, Russia

E-mail address: tak@san.ru 


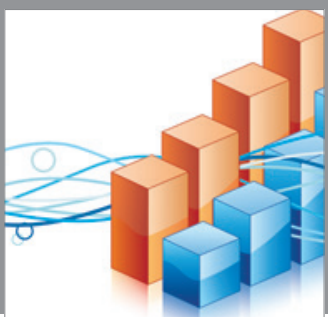

Advances in

Operations Research

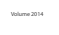

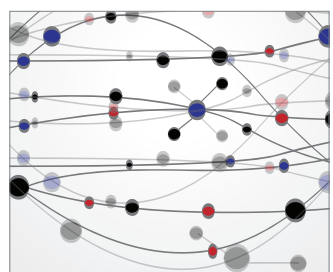

\section{The Scientific} World Journal
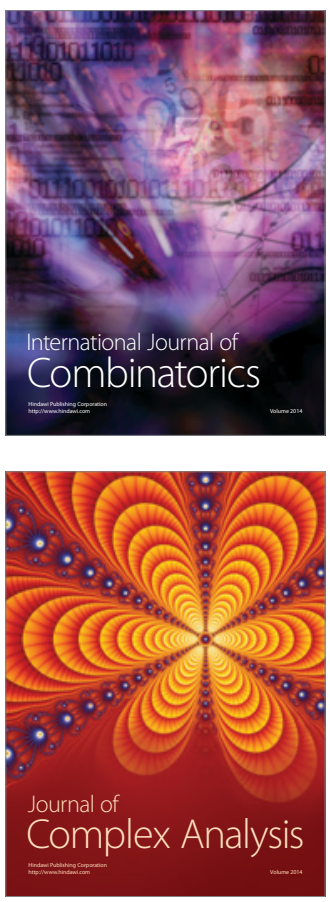

International Journal of

Mathematics and

Mathematical

Sciences
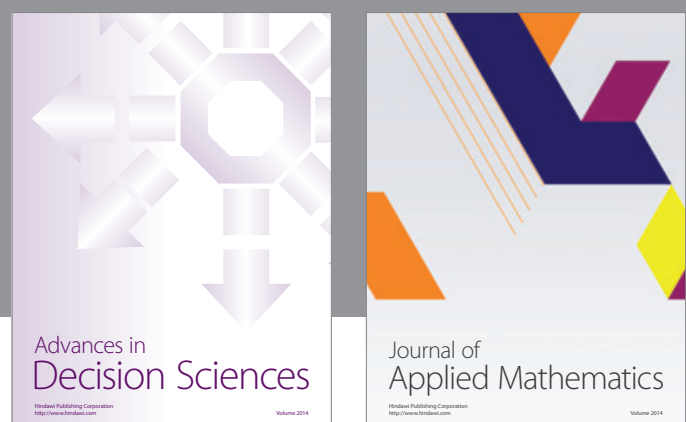

Journal of

Applied Mathematics
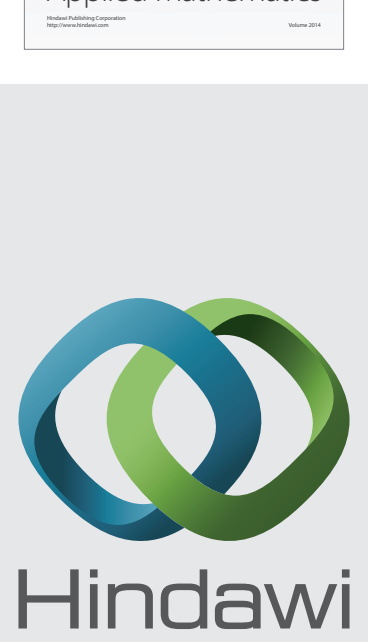

Submit your manuscripts at http://www.hindawi.com
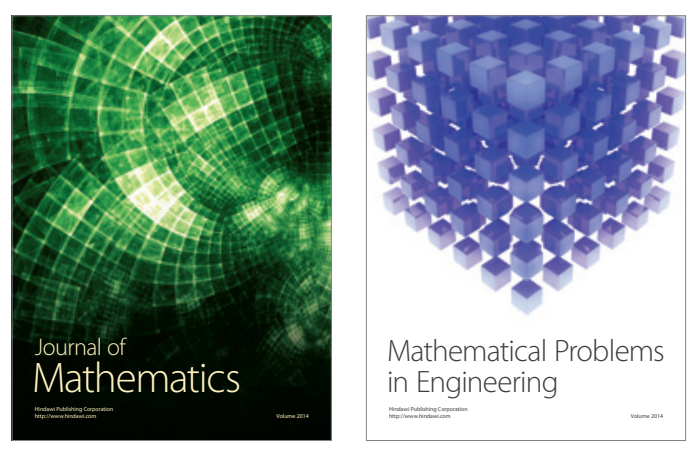

Mathematical Problems in Engineering
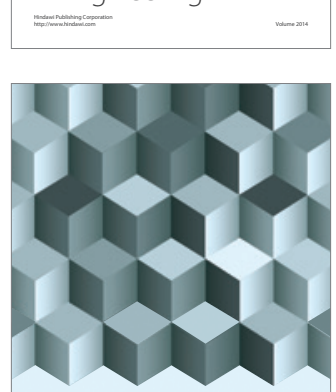

Journal of

Function Spaces
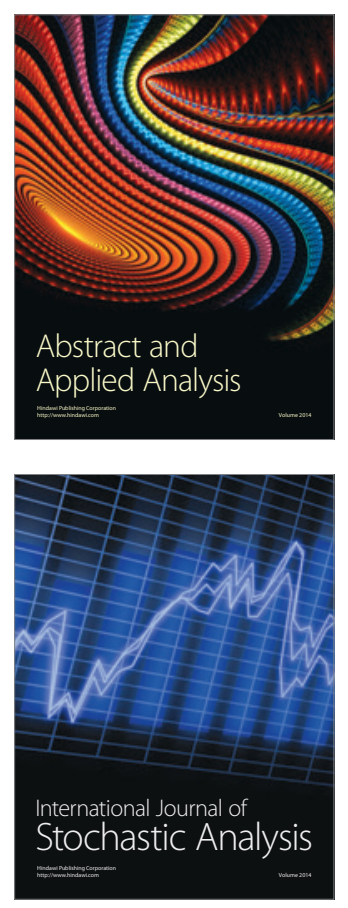

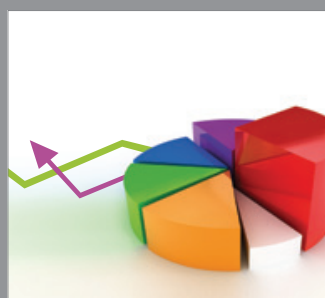

ournal of

Probability and Statistics

Promensencen
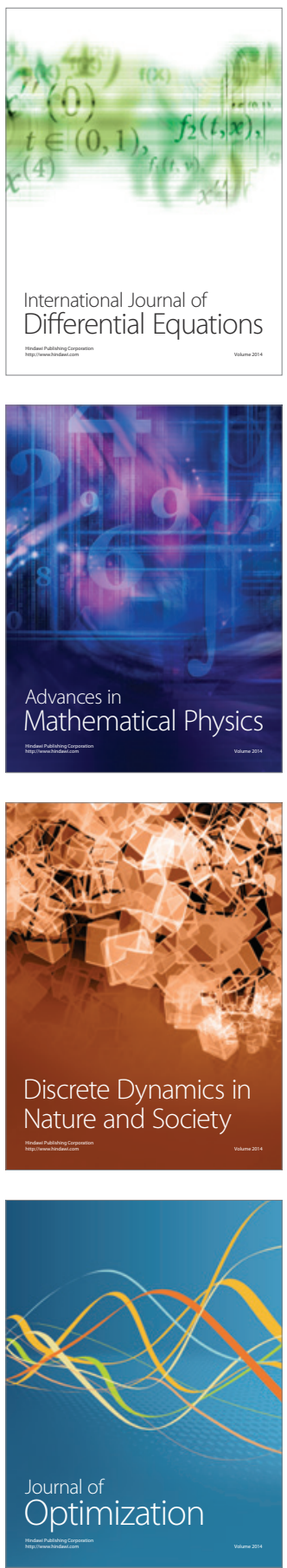